\title{
New reactor dedicated to in operando studies of model catalysts by means of surface x-ray diffraction and grazing incidence small angle $x$-ray scattering
}

\author{
M.-C. Saint-Lager, a) A. Bailly, ${ }^{\text {b) }}$ P. Dolle, R. Baudoing-Savois, P. Taunier, S. Garaudée, \\ S. Cuccaro, S. Douillet, O. Geaymond, G. Perroux, and O. Tissot \\ Institut Néel, CNRS and Université Joseph Fourier, BP 166, F-38042 Grenoble Cedex 9, France \\ J.-S. Micha, O. Ulrich, and F. Rieutord \\ CEA/DRFMC, 17 rue des Martyrs, 38054 Grenoble Cedex 9, France
}

(Received 11 April 2007; accepted 29 June 2007; published online 9 August 2007)

\begin{abstract}
A new experimental setup has been developed to enable in situ studies of catalyst surfaces during chemical reactions by means of surface $\mathrm{x}$-ray diffraction (SXRD) and grazing incidence small angle $\mathrm{x}$-ray scattering. The $\mathrm{x}$-ray reactor chamber was designed for both ultrahigh-vacuum (UHV) and reactive gas environments. A laser beam heating of the sample was implemented; the sample temperature reaches $1100 \mathrm{~K}$ in UHV and $600 \mathrm{~K}$ in the presence of reactive gases. The reactor equipment allows dynamical observations of the surface with various, perfectly mixed gases at controlled partial pressures. It can run in two modes: as a bath reactor in the pressure range of 1-1000 mbars and as a continuous flow cell for pressure lower than $10^{-3}$ mbar. The reactor is connected to an UHV preparation chamber also equipped with low energy electron diffraction and Auger spectroscopy. This setup is thus perfectly well suited to extend in situ studies to more complex surfaces, such as epitaxial films or supported nanoparticles. It offers the possibility to follow the chemically induced changes of the morphology, the structure, the composition, and growth processes of the model catalyst surface during exposure to reactive gases. As an example the $\mathrm{Pd}_{8} \mathrm{Ni}_{92}(110)$ surface structure was followed by SXRD under a few millibars of hydrogen and during butadiene hydrogenation while the reaction was monitored by quadrupole mass spectrometry. This experiment evidenced the great sensitivity of the diffracted intensity to the subtle interaction between the surface atoms and the gas molecules. () 2007 American Institute of Physics.
\end{abstract}

[DOI: $10.1063 / 1.2766821]$

\section{INTRODUCTION}

In the field of catalysis, surface science aims to better understand of the catalytic process at both the atomic and molecular scales. By contrast to industrial applications or other research approaches where microporous catalysts or powders of supported nanoparticles are used, surface science deals with model catalysts such as extended flat surfaces of single crystals elaborated in ultrahigh-vacuum (UHV) environment. However, more recently, studying systems closer to real catalysts and in (semi-) realistic reaction conditions is becoming a very active research field of surface science. Particularly, many efforts are done to bridge the so-called "pressure and material gaps," experimentally but also theoretically. Regarding this last point, the most common approach uses first-principles atomistic total energy combined with gas phase thermodynamics. ${ }^{1,2}$ The atomic structure predicted by minimizing the total energy of the surface exposed to a gas phase at high pressure can be quite different from the one at low pressure. On the experimental side many developments

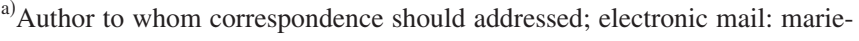
claire.saint-lager@grenoble.cnrs.fr

${ }^{b)}$ Present address: CEA-LETI, Minatec, 17 rue des Martyrs, 38054 Grenoble Cedex 09, France.
}

have been done to directly monitor the surface during catalytic reaction. New surface science tools must be conceived and designed to be compatible with the high pressure of reactive gases. Some standard surface science probes are in first view ruled out, particularly those which strongly interact with matter. Even though sophisticated devices involving differentially pumped pressure cells allow to perform x-ray photoemission, electron microscopy, low energy ion scattering, and soft x-ray absorption in high pressure conditions, some other techniques are much more evident such as scanning tunnelling microscopy (STM) even if in situ measurements need well-adapted instruments. ${ }^{1}$ Other techniques dealing more with the interaction of molecules with surfaces such as polarized reflection absorption infrared spectroscopy $^{3}$ (RAIRS) and sum frequency generation ${ }^{4}$ (SFG).

Actually one of the most promising techniques for in situ structural investigations of surfaces is surface x-ray diffraction (SXRD) which is, compared to low energy electron diffraction (LEED), not restricted to low pressure environments nor to noninsulating samples. The original setup devoted to in situ measurements of solid/gas interfaces by SXRD was built on the ID03 beamline at the European Synchrotron Radiation Facility (ESRF). ${ }^{5}$ Thanks to this instrument, a strong 
temperature-dependant structural change of the $\mathrm{Ni}(110)$ surface was evidenced in the presence of a few millibars of carbon monoxide $(\mathrm{CO})$. At room temperature, the high pressure and UHV structures are identical, whereas at $400 \mathrm{~K}$, the $\mathrm{CO}$ high pressure induces a restructuring of the Ni substrate that develops strained (111) microfacets. ${ }^{6}$ The modifications of the $\mathrm{Pd}_{8} \mathrm{Ni}_{92}(110)$ surface under butadiene and hydrogen, as well as during butadiene hydrogenation at elevated pressure, at $300 \mathrm{~K}$, were also studied with this high pressure chamber. ${ }^{7}$ The $(N \times 1)$ reconstruction was found to be still present. The main changes were observed for a surface peak intensity under pure hydrogen and during butadiene hydrogenation. Another experiment was devoted to the structural characterization of the surface oxide during $\mathrm{CO}$ oxidation on $\mathrm{Pt}(110){ }^{8}$ This work illustrated the good complementarity between in situ SXRD and STM. Morphology changes of the surface during $\mathrm{CO}$ oxidation corresponding to the activity cycle were first evidenced by STM (Ref. 9) and were then correlated to the structural changes by SXRD. These experiments allowed to clearly show that the oxide surface is the more active.

The purpose of this new experimental setup was to extend SXRD studies to more complex surfaces such as thin epitaxial films or supported nanoparticles. This needs to connect the x-ray reactor to an auxiliary UHV chamber to prepare these complex surfaces just before the $\mathrm{x}$-ray experiment. In addition such a chamber can be equipped with Auger electron spectroscopy (AES) allowing to also chemically probe the surface.

For supported nanoparticles, information about their morphology (size, shape, cluster density, etc.) are crucial and a particularly relevant technique is grazing incidence small angle x-ray scattering (GISAXS). One of the first experiments was done on gold aggregates deposited on a flat surface of silicon. ${ }^{10}$ In situ measurements during Pd nanoparticle growth on $\mathrm{MgO}(100)$ (Ref. 11) show how GISAXS can be very promising for in situ catalytic reactions. Several works report in situ GISAXS during nanoparticle growth for catalysis but in UHV conditions. ${ }^{12}$ Very recently the thermal stability of supported platinum clusters was studied by in situ GISAXS in UHV and in the presence of hydrogen, ${ }^{13}$ but none of them concerns measurements under pressures corresponding to realistic reaction conditions.

For well organized supported nanoparticles the same information, added to the structure, can be extracted from SXRD. Such a study was performed during the oxidation of Pd nanoparticules on $\mathrm{MgO}(100) .{ }^{14}$ In this work, the sample was not in situ prepared and was thus exposed to air before the SXRD measurements.

This article is divided into two main parts. First, a detailed description of the setup is given. The second one presents the first experimental results it allowed to obtain by SXRD during butadiene hydrogenation on $\mathrm{Pd}_{8} \mathrm{Ni}_{92}(110)$.

\section{DESIGN OF THE EXPERIMENTAL SETUP}

\section{A. Global requirements}

The aim of this new experimental setup is thus to study model catalysts which are prepared under UHV environment

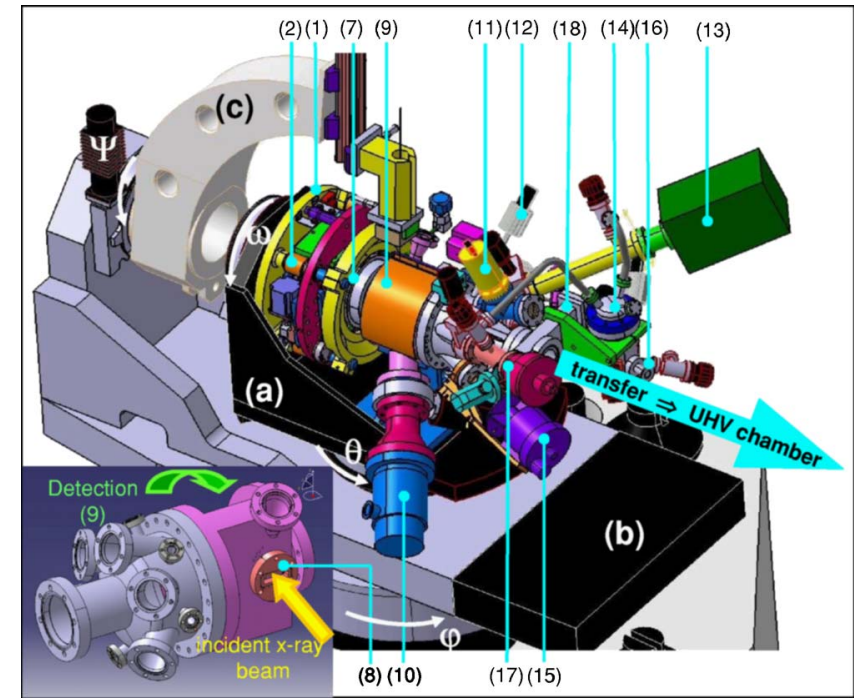

FIG. 1. (Color online) Overview of the reactor mounted on the GMT diffractometer. The reactor is set on the $\theta$ table (a), (b) is the $\phi$ detector table and (c) is the $\psi$ detector arm. The reactor equipments are detailed in the text. The large arrow indicates the UHV transfer to the preparation chamber.

and then analyzed by SXRD or GISAXS under catalytic reaction conditions. To meet this purpose a geometry with two horizontally connected chambers was chosen: the x-ray reactor chamber and an UHV chamber. The sample is prepared and characterized in the UHV chamber. It is then transferred under UHV conditions in the x-ray reactor, designed for both UHV and reactive gas environments to perform in operando studies. This latter point means that a special attention was paid to the choice of the materials inside the reactor.

The whole setup was designed to be mounted on the "Goniomètre Multi-Techniques" (GMT) of the French BM32 beamline at ESRF. ${ }^{15}$ This diffractometer is a versatile instrument dedicated to surface and interface studies. Although it can handle heavy and large experimental setups, the available space is limited. Another feature of the setup is the possibility of easily moving it between the laboratory and synchrotron environments allowing classical studies (sample preparation and reaction conditions) prior to allocated beam time at ESRF.

\section{B. Goniometer head and coupling to the GMT diffractometer}

The labels used in the following description refer to Figs. 1-3. The design of the whole setup is represented in Fig. 1. The GMT diffractometer geometry is of the " $2+2$ " type. ${ }^{16}$ The sample surface is held in a vertical position; $\theta$ defines the polar incident beam angle and $\omega$, the azimuthal rotation. The detector motions are $\varphi$ for the out-of-plane rotation and $\psi$ for the in-plane one.

SXRD and GISAXS require a grazing incidence angle together with a highly accurate positioning of the sample surface inside the incident $\mathrm{x}$-ray beam. This is ensured by a goniometer head with three degrees of freedom (see Fig. 2): two crossed tilts, $\chi_{1}$ and $\chi_{2}$, with perpendicular rotation axes, which allow the alignment of the surface sample normal with the axis of the $\omega$ rotation, and the $Z$ translation to put the surface sample at the diffractometer homocenter. 


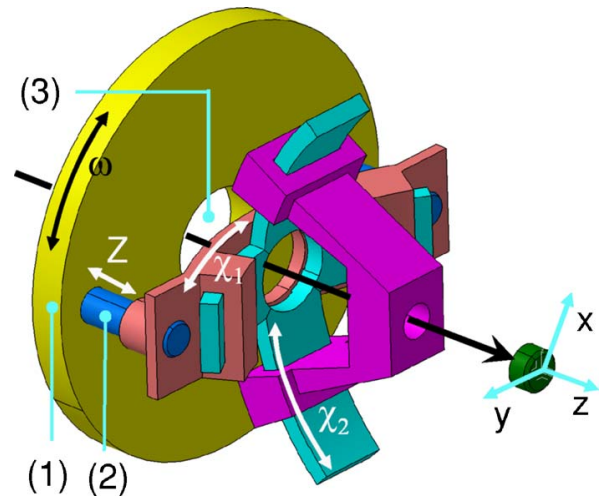

FIG. 2. (Color online) The three degrees of freedom of the goniometer head: $\chi_{1}, \chi_{2}$, and $Z$. The relative scales are not respected to evidence the assembly principle. The straight black arrow indicates the laser beam path.

The whole mechanics is held on the large circular flange (1) fixed on the $\omega$ circle of the GMT diffractometer. The $Z$ translation is guided by four prestressed columns (2) and geared by one motor and two screws; its amplitude is $\pm 2 \mathrm{~mm}$ with a twist less than $0.01^{\circ}$. The $Z$ positioning accuracy and reproductiveness are $\pm 1 \mu \mathrm{m}$, controlled by an encoder (Renishaw $\mathrm{RGH} 24 \mathrm{~W})$ with $0.2 \mu \mathrm{m}$ resolution. The two rocker cradles of the $\chi_{1}$ and $\chi_{2}$ rotations are also represented in Fig. 2. Their amplitude is $\pm 2^{\circ}$ with a confusion circle diameter less than $2 \mu \mathrm{m}$. Figure 4 illustrates the accuracy and the reproducibility of these motions as record with $\mathrm{TiO}_{2}$ surface oxide in the direct beam for the $Z$ translation [Fig. 4(a)] and on the reflectivity for the two $\chi_{1}$ and $\chi_{2}$ rotations [Figs. 4(b) and $4(\mathrm{c})$, respectively].

As shown on the section of the goniometer head and of the reactor chamber (Fig. 3), the design is very compact fitting into the limited available space of the $\theta$ table [label (a) in Fig. 1]. The distances between the diffractometer homocenter are $275 \mathrm{~mm}$ to the vertical support and $252 \mathrm{~mm}$ to the horizontal platform. The whole mechanics stands within a cylinder $\left(D \times H=400 \times 100 \mathrm{~mm}^{2}\right)$ offering a free axial cylindrical hole of $120 \mathrm{~mm}$ diameter (3). This available space inside the mechanics is occupied by an extension of the re-

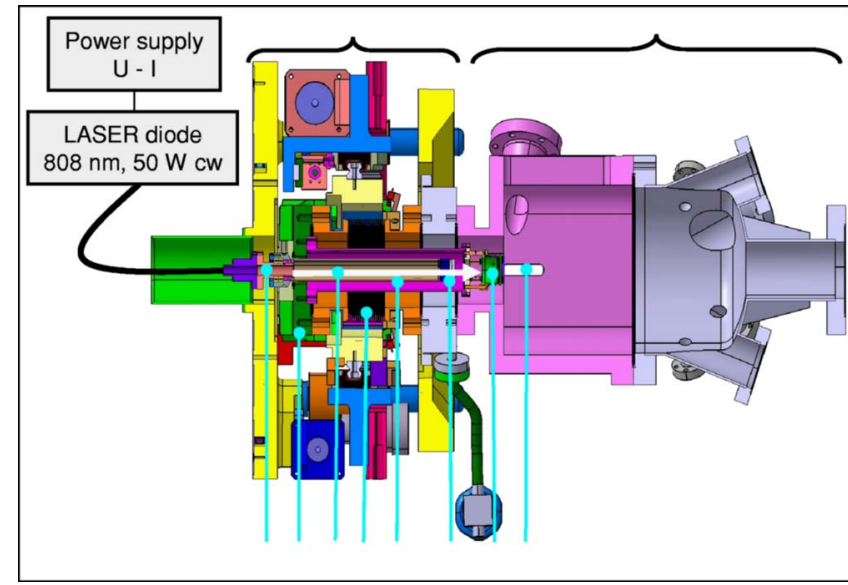

FIG. 4. (Color online) (a) Intensity of the x-ray direct beam recorded during the $Z$ translation of the $\mathrm{TiO}_{2}$ sample after its surface normal was aligned with the $\omega$ of the diffractometer. The working position is defined when the intensity is divided by two. Between the two curves, several motions were performed; $\omega, \chi_{1}$, and $\chi_{2}$ rotations, and $Z$ translation. The shift between the two curves is less than $2 \mu \mathrm{m}$, showing the high accuracy and the reproductiveness of the motions. [(b) and (c)] $\chi_{1}$ and $\chi_{2}$ variations on the $\mathrm{TiO}_{2}$ reflectivity taken around an incident angle two times the critical angle $\left(0.13^{\circ}\right.$ at $18 \mathrm{keV}$ ). As for (a) several motions separated the two curves on each figure and their discrepancy is less than $5^{\circ} / 1000^{\circ}$.

actor chamber made of a bellows (4). The end of this extension is fixed on the goniometer head by a welded flange (5) which moves with the $\chi_{1}, \chi_{2}, Z$, and $\omega$ motions and onto which the sample holder (6) is mounted. This bellows (4) allows for the $\chi_{1}, \chi_{2}$, and $Z$ motions of the sample holder inside the main volume of the reactor, which is fixed onto the $\theta$ circle of the GMT diffractometer. The coupling between the movable extension, including the sample holder (6), and the fixed part of the reactor chamber is made by a CF63 RNN-250/3 differentially pumped rotary platform from Thermionics (7) enabling the $\omega$ rotation $\left(360^{\circ}\right)$.

The movable part of the reactor chamber can be baked with a heating removable device installed, at the atmosphere, in a tube (21) within the sample holder. A stainless steel screen surrounds the bellows (4) to protect the mechanics

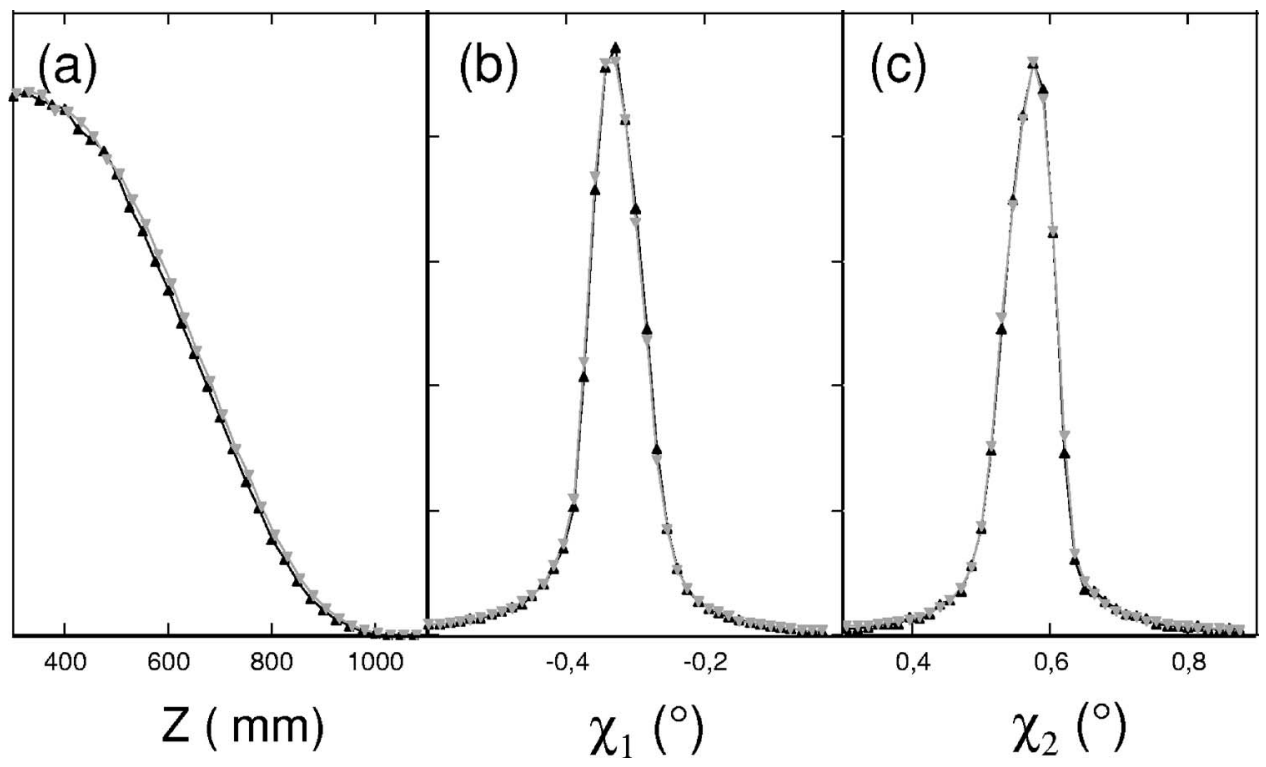

FIG. 3. Vertical cross section of the mechanics and of the reactor. The laser beam device used for the sample heating is also shown. 
during the bakeout. In addition zirconium oxide insulators are inserted between the end flange (5) of the reactor and its mounting on the mechanics. Two thermocouples are also set in the mechanics to monitor its temperature during the bakeout.

\section{The reactor chamber}

The reactor, in which the sample is studied under reactive conditions with the synchrotron beam, is made of stainless steel. For clarity, it can be divided into three main parts. The first is the movable part described just above. It includes the sample holder and its heating device (discussed in Part Sec. II D).

The second part is the main body of the chamber: it is equipped with two beryllium windows (Brushwellman company) for the incoming and scattering $\mathrm{x}$-ray beams. The reactor shape is a cylinder truncated on the side of the incident $\mathrm{x}$-ray beam (see insert in Fig. 1 and the cross section in Fig. 3). This was especially designed to be compatible with GISAXS and to allow the addition of antiscattering slits. The entrance beryllium window is mounted on a CF40 port (8). The large exit beryllium window (9) is welded on the cylindrical part of the reactor chamber (200 $\mathrm{mm}$ diameter) allowing the detection of scattered beams from $-1^{\circ}$ up to $95^{\circ}$ in the in-plane direction $(\psi)$ and from $-1^{\circ}$ up to $40^{\circ}$ in the out-of-plane one $(\phi)$.

The third part is equipped with several ports for the reactor equipment.

- A turbomolecular pump (10), protected by an automated butterfly valve, is installed on a CF63 port for pumping down to UHV (pumping speed: $210 \mathrm{l} / \mathrm{s}$ for $N_{2}$ ).

- Three pressure gauges allow the pressure monitoring from $10^{-10}$ mbar up to 1 bar: a cold cathode gauge (11) for UHV and two capacitance diaphragm gauges (12) for $10^{-4}$ up to 1 mbar and for 1 up to 1000 mbars.

- The gas composition in the reactor can be monitored by a quadrupole mass spectrometer (Prisma ${ }^{\mathrm{TM}}$ QMS $200 \mathrm{M} 1$ of Pfeiffer) (13) mounted behind a CF40 gate valve. For pressures higher than $10^{-6} \mathrm{mbar}$, the reactants and products are sampled through a leak valve.

- A subsidiary turbomolecular pump (14) is hung on the reactor chamber (pumping speed: $60 \mathrm{l} / \mathrm{s}$ for $N_{2}$ ) and coupled to an UHV gauge. It supplies pumping for the equipments which must not be exposed to a high pressure of gases such as the mass spectrometer and the ion sputtering gun.

- The sample temperature is measured with an external infrared pyrometer (15) (Impac IPE 140 MB 10) pointing to the sample surface through a sapphire view port. The spectral band is $3-5 \mu \mathrm{m}$ and the nominal temperature range is $300-1300 \mathrm{~K}$ with $0.1 \mathrm{~K}$ resolution for the emissivity $\varepsilon$ ranging from 0.1 to 1 . The minimum readable temperature depends on the emissivity: $300 \mathrm{~K}$ for $\varepsilon=1$ and $350 \mathrm{~K}$ for $\varepsilon=0.1$.

- Two view ports allow one to look inside the reactor. They are localized in the vertical plane, symmetric relative to the sample surface normal, and are used for laser alignment of the normal along the $\omega$ axis.
- The gas input (16) is isolated by an electropneumatic CF16 gate valve in line with a stainless steel microvalve and a 0.61 reservoir with a capacitive pressure gauge $\left(10^{-4}-1 \mathrm{mbar}\right)$. This setting allows two operation modes:

Fast expansion of the high pressure of a reactive mixture by the remote opening of the electropneumatic valve. The surface diffraction and gas analyses are thus performed instantaneously and simultaneously just after the expansion and then followed in a static mode. The reactor turbomolecular pump has been previously isolated from the reactor chamber by closing its electropneumatic CF63 butterfly valve. The two electropneumatic valves are controlled by a pilot regulated by the chamber and the reservoir pressures. In this configuration the microvalve acts to diminish the speed of the gas flux to avoid a shock wave in the reactor during the expansion.

(ii) Low pressure gas exposures $\left(<10^{-3} \mathrm{mbar}\right)$ are made in a dynamical mode: the two electropneumatic valves are opened and the gas flux is manually regulated by the microvalve aperture.

The reservoir is connected to the gas manifold where gases and the reactive mixture are stored before being introduced in the reactor. It is equipped with a 1-1000 mbars capacitive pressure gauge. The manifold is pumped with an ancillary pumping station (primary and turbomolecular pumps). This station can also be connected to the reactor for rough pumping after gas exposures at pressure close to the atmosphere.

- An ion sputtering gun (17) can be mounted behind a CF40 gate valve ensuring its isolation during gas exposures. It is also connected to the subsidiary turbomolecular pump (14) via a right-angle CF16 valve. When the ion gun is mounted on the reactor it allows the preparation of single crystal surfaces in UHV environment, directly in the reactor, without any transfer to the preparation chamber.

The reactor ends with a CF63 gate valve (18) to connect it to the UHV chamber. The reactor total volume is approximatively 5.51 .

\section{Sample heating}

The sample is heated by a laser beam as first done by Kuhrs and et al. ${ }^{17}$ A high-power fiber-coupled laser diode (Jenoptik JOLD-50-CPXI) generates a $808 \mathrm{~nm}$ beam with a power of up to $50 \mathrm{~W}$. The external power supply (Delta Electronika SM 156100) delivers an operating current up to 45 A corresponding to the maximum heating power. The stable working temperature of the diode is achieved by water cooling.

The scheme of Fig. 3 shows the laser heating setup. The laser beam is guided up to the reactor by a multimode fiber (600 $\mu \mathrm{m}$ diameter) ended by an SMA connector with an optical aperture of 0.22 (corresponding to the sine of the aperture angle). The collimation is achieved by an optical system (19) chosen to give a spot with a diameter of $10-11 \mathrm{~mm}$ at the sample position. This collimation optics is held at the entrance of the mechanics; a ball bearing coupling avoids 


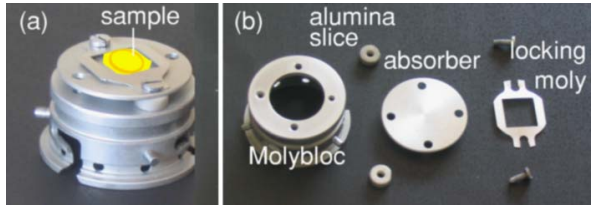

FIG. 5. (Color online) Pictures of (a) the sample mounted on a Molybloc and (b) the different parts needed for mounting the sample on the Molybloc.

twisting of the optical fiber during azimuthal rotation of the goniometer head. The whole optical system and the fiber can be removed during the reactor bakeout. The collimated beam (20) enters a stainless steel tube (21) supported by the reactor end flange (5). The other end of this tube is welded to a silica window and it ensures the reactor airtightness. Even if the silica reflectivity is low for the laser wavelength, it is not negligible and for the maximum diode power the reflected part can reach almost $4 \mathrm{~W}$. To avoid damages of the diode by the power reflected back in the fiber, the silica window is tilted $5^{\circ}$ relative to the laser beam. It is surrounded, inside the reactor, by a concentric cylinder (6) ended by a support which is fixed on an alumina thermal insulating ring. On this support, sample holder (Molybloc) (22) can be locked with a mechanical transfer rod. The sample mounting on the Molybloc is shown on Fig. 5.

Compared to the setup of Kuhrs et al., ${ }^{17}$ only one $50 \mathrm{~W}$ laser diode was used but the efficiency of the heating was improved. The most common metals used in catalysis are, for example, platinum or palladium, which have a very high reflectivity in the near infrared region. For this reason, most of the laser beam is lost by backreflection. An absorber was thus inserted on the path of the laser beam, in close contact with the sample in order to heat it by thermal conduction. For catalytic reactions involving hydrogen and hydrocarbons, a molybdenum piece can be used to absorb the incident laser power. Nevertheless, the Mo reflectivity, even for a rough surface, is still equal to 0.55 at $808 \mathrm{~nm}$. Darkening the absorber was not enough to make it as close as possible to a blackbody. The absorption was thus made more efficient thanks to tilted ribs machined on the laser beam illuminated side of the absorber (see Fig. 6). The rib geometry was optimized in order to trap most of the photons by way of a multiple-reflection process (at least two reflections). The laser diode power supply can be controlled by a PID pilot regulated by the sample temperature measured by the IR pyrometer.

The performance of this laser heating was checked by measuring the temperature on the absorber and on the sample. The emissivity used for the IR pyrometer was calibrated with the help of a thermocouple provisionally installed at the end of a wobble stick. As shown in Fig. 7, the sample surface temperature can reach $1100 \mathrm{~K}$ in UHV. In reactive gas environment the maximum temperature is limited to $600 \mathrm{~K}$. It is limited by the thermal exchange with the air. But also because above this temperature the interaction of the molecules with the reactor walls and the sample holder becomes non-negligible compared to the sample surface activity.

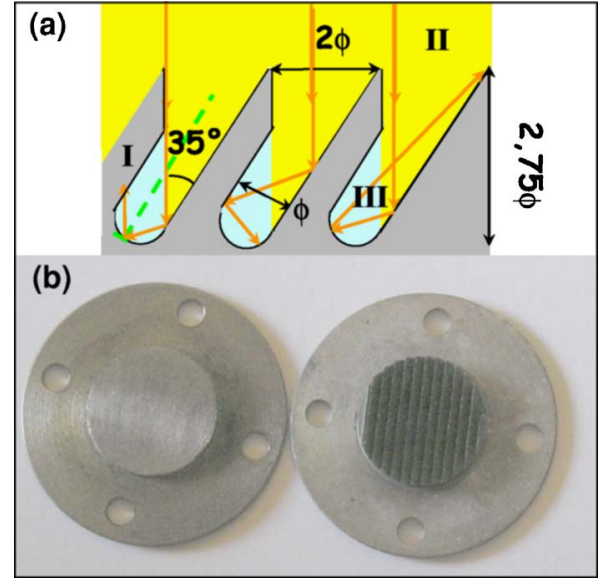

FIG. 6. (Color online) (a)Scheme of photon trapping between the ribs (I), machined in the laser beam illuminated side of the absorber. Each photon of the laser beam (II) is reflected at least two times before escaping from the trap, region III is the space between the ribs where the laser beam goes after the first reflection. (b) Top view of the machined Mo absorber (on the right) with a width $2 \phi$ between two ribs equal to $1 \mathrm{~mm}$, compared to an Mo flat surface (on the left). At normal incidence the machined surface looks darker than the flat one.

\section{E. The UHV preparation chamber}

The UHV preparation chamber is connected to the reactor by a communication UHV chamber through which the sample mounted on its Molybloc (Fig. 5) moved with a mechanical transfer rod. The UHV chamber is also equipped with a small ancillary load-lock chamber for extracting the sample of the setup back to air and installing an another one. The pumping is achieved with turbomolecular, ionic, and titanium sublimation pumps.

There are two working levels separated by $136 \mathrm{~mm}$ height: (i) the transfer level and (ii) the preparation and characterization level with an electron bombardment source and a Knudsen-type evaporator for deposition, a retractable quartz microbalance to calibrate the flux, a retractable LEED/AES (Omicron NanoTechnology), an electron gun for medium energy electron diffraction (MEED), and an ion sputtering gun (argon and oxygen ions). In this chamber the

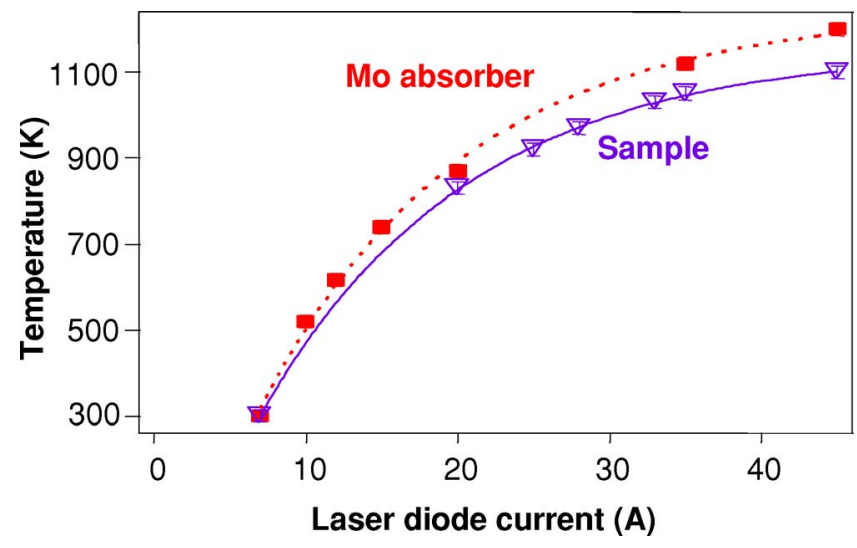

FIG. 7. (Color online) Temperature on the Mo absorber and on the surface sample in UHV as a function of the current supplied to the laser diodes. The measurement was done with a thermocouple mounted on a wobble stick for the Mo absorber and with the IR pyrometer for the sample. In both cases the errors bars are inside the symbols. 
Molybloc with the sample is locked on an oven with heating by electron bombardment.

The sample holder is mounted on a manipulator with two perpendicular horizontal translations, and a vertical translation (parallel to the surface sample), plus a rotation around the vertical axis. Several view ports are also installed, two of them are at an angle of $55^{\circ}$ symmetric relative to the surface normal to offer the possibility of performing surface differential reflectivity spectroscopy (SDRS).

\section{FIRST RESULTS AND PERFORMANCE: SURFACE $X$-RAY DIFFRACTION DURING BUTADIENE HYDROGENATION ON $\mathrm{Pd}_{8} \mathrm{Ni}_{92}(110)$}

To assess the performance of this setup, SXRD was performed during butadiene hydrogenation on the (110) surface of the $\mathrm{Pd}_{8} \mathrm{Ni}_{92}$ alloy. The ion gun was installed on the reactor chamber to clean the surface sample and the UHV preparation chamber was not necessary. First of all we checked that without the sample no reaction occurs in the reactor chamber.

\section{A. Previous results}

This study was the continuation of a published work. ${ }^{7}$ In this article we showed that the $(N \times 1)$ reconstruction $(N$ =5-6) observed for the clean $\mathrm{Pd}_{8} \mathrm{Ni}_{92}(110)$ consits of undulating outer dense rows, constituted mainly of $\mathrm{Pd}$ atoms which segregate to the surface. In the alloy surface plane, the $\mathrm{Pd}-\mathrm{Pd}$ interatomic distances are shorter than in pure palladium. On the contrary the first interlayer spacing is larger. This reconstruction allows partial relaxation, of the strain due to the $10 \%$ lattice mismatch between $\mathrm{Pd}$ and $\mathrm{Ni}$ atoms.

The $(N \times 1)$ surface reconstruction was still visible in the presence of butadiene, hydrogen, and even during the butadiene hydrogenation performed under elevated pressure (5-50 mbars) at $300 \mathrm{~K}$. The main modifications concerned the surface diffraction peak intensities. A large and fast decrease of the $\left(\begin{array}{lll}0 & 1 & 0.028\end{array}\right)$ surface peak intensity was observed at the beginning of the butadiene hydrogenation reaction (0.5 mbar butadiene and 5 mbar hydrogen). Afterwards the intensity of this surface diffraction peak gently evolved during the course of the reaction correlative to the two steps of the reaction. It turned out that the large intensity decrease at the beginning of the reaction was the same as that observed under 5 mbars, of pure hydrogen. Moreover a similar behav-

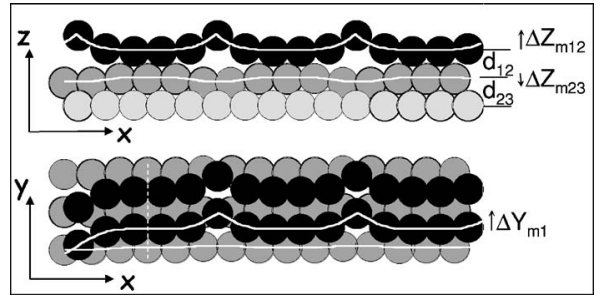

FIG. 8. Side and top views of the structure of the clean $\operatorname{Pd}_{8} \mathrm{Ni}_{92}(110)(5$ $X 1)$ surface as deduced from surface $X$-ray diffraction data in Ref. 7. $X$ and $Y$ are in the surface plane ( $X$ corresponding to the dense rows [1 $\overline{10}]$ ) and $Z$ is perpendicular to the surface. The dark circles represent surface $\mathrm{Pd}$ atoms and the light ones the bulk (Pd or Ni) atoms.

ior for the (01) rod in the presence of pure hydrogen and during butadiene hydrogenation experiments was observed. This led us to conclude that the role of hydrogen was essential during the reaction. But, as pointed out in Ref. 7, this was in apparent contradiction with generally admitted concepts issued from the kinetics of this reaction on Pd-based catalysts. Thus, the aim of this new experiment was (i) to reproduce the previous results obtained with the highpressure chamber of ID03 (Ref. 5) to check the new setup and (ii) to extend the data collection over additional points in the reciprocal space to better understand the respective role of each component of the reactive gas mixture on the structural changes.

\section{B. UHV results}

The $\mathrm{Pd}_{8} \mathrm{Ni}_{92}(110)$ surface was prepared by a series of $\mathrm{Ar}^{+}$sputtering and annealing at $1000 \mathrm{~K}$ until an $(N \times 1)$ reconstruction similar to that already observed ${ }^{7}$ was obtained. The SXRD data were recorded at a beam energy of $18 \mathrm{keV}$, at grazing incidence $\left(0.2^{\circ}\right)$. Measurements were first performed under UHV in a base pressure of $2.10^{-9}$ mbar. The $(N \times 1)$ reconstruction model, sketched on Fig. 8, was used to fit the data along the (10), (01), (11), and (02) crystal truncation rods (CTRs) and their equivalents. As shown in Table I, the geometrical parameters were found very close to those previously reported. ${ }^{7}$

\section{Results under high pressure of hydrogen and under reaction conditions}

The surface was then exposed to 10 mbars of hydrogen (N55 gas purity) and the intensities at (llll 10.03$)$ and $\left(\begin{array}{ll}0 & 1\end{array}\right.$

TABLE I. Structural parameters obtained for the $\mathrm{Pd}_{8} \mathrm{Ni}_{92}(110)$ reconstruction, as checked in Fig. 8 and obtained by fitting SXRD data. Three different sets of values are reported: in UHV from Ref. 7, in UHV with the present experimental setup and this work under 10 mbars of hydrogen. $\beta$ is the surface roughness, $c_{1}$ and $c_{2}$ are the Pd concentrations in the surface layers 1 and 2, respectively, $\Delta d_{12}$ is the global shift of interlayer distance between the first and the second atomic layers relative to the bulk reference, $\Delta d_{23}$ is the same for layers 2 and $3, \Delta Y_{m 1}$ is the amplitude of the in-plane row oscillation for the first layer, $\Delta Z_{m 12}$ is the amplitude of the out-of-plane row oscillation for the first layer, and $\Delta Z_{m 23}$ is amplitude of the out-of-plane row oscillation for the second layer.

\begin{tabular}{ccccccccc}
$\exp$ & $\beta$ & $c_{1}$ & $c_{2}$ & $\Delta d_{12}$ & $\Delta d_{23}$ & $\Delta Y_{m 12}$ & $\Delta Z_{m 12}$ & $\Delta Z_{m 23}$ \\
\hline $\mathrm{UHV}^{\mathrm{a}}$ & 0.34 & 1 & 0.1 & 0.057 & 0.00 & 0.42 & 0.32 & -0.1 \\
$\mathrm{UHV}$ & 0.31 & 1 & 0.1 & 0.052 & 0.046 & 0.35 & 0.35 & -0.2 \\
$\mathrm{~h} 2$ & 0.31 & 1 & 0.1 & 0.063 & 0.042 & 0.5 & 0.3 & -0.2 \\
\hline \hline
\end{tabular}

${ }^{\mathrm{a}}$ Reference 7 . 


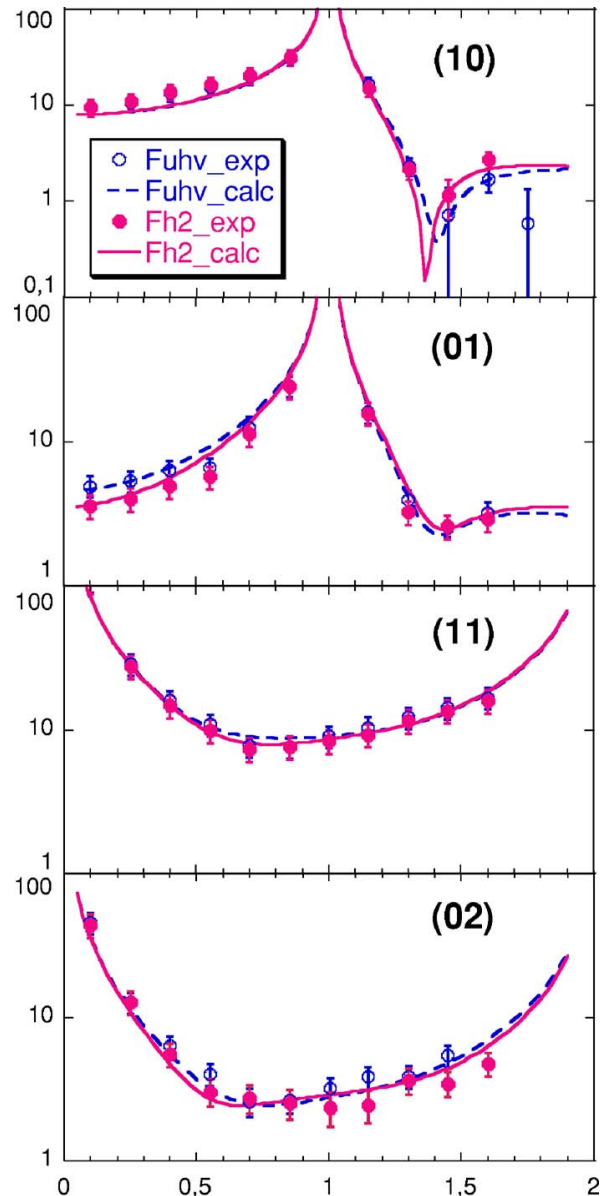

FIG. 9. (Color online) Comparison between the experimental (circles) and the calculated (line) structure factors along the (10), (01), (11), and (02) CTRs. Empty circles are for UHV measurements and filled ones are for

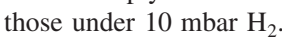

0.03 ) were followed for several hours. The first one did not vary, while the second dropped at the very beginning of the exposure and then kept a constant value. Interestingly, it recovered its initial intensity after pumping hydrogen. As the diffracted intensities are stable during the hydrogen exposure it was possible to record the (10), (01), (11), and (02) CTRs and their equivalents under 10 mbar hydrogen in order to compare the structure to the UHV one. Figure 9 shows the experimental structure factor together with the calculated ones. The same model as in UHV was used to fit the data recorded under hydrogen pressure but with different parameters as shown in Table I. The two main differences concern (i) the expansion of the first interlayer spacing indicating the presence of subsurface hydrogen and (ii) an enhancement of the amplitude of the surface dense rows modulation in the $Y$ direction.

The surface was finally studied under 5 mbars of a butadiene/hydrogen mixture with the ratio 1:8 (N25 gas purity for butadiene). Figure 10(a) shows the evolution of the partial pressures of butadiene, butenes, and butane in the reactor. With the reaction is totally selective with respect to butenes during the whole time of butadiene conversion. The reactor volume being large, the reaction rate was initially

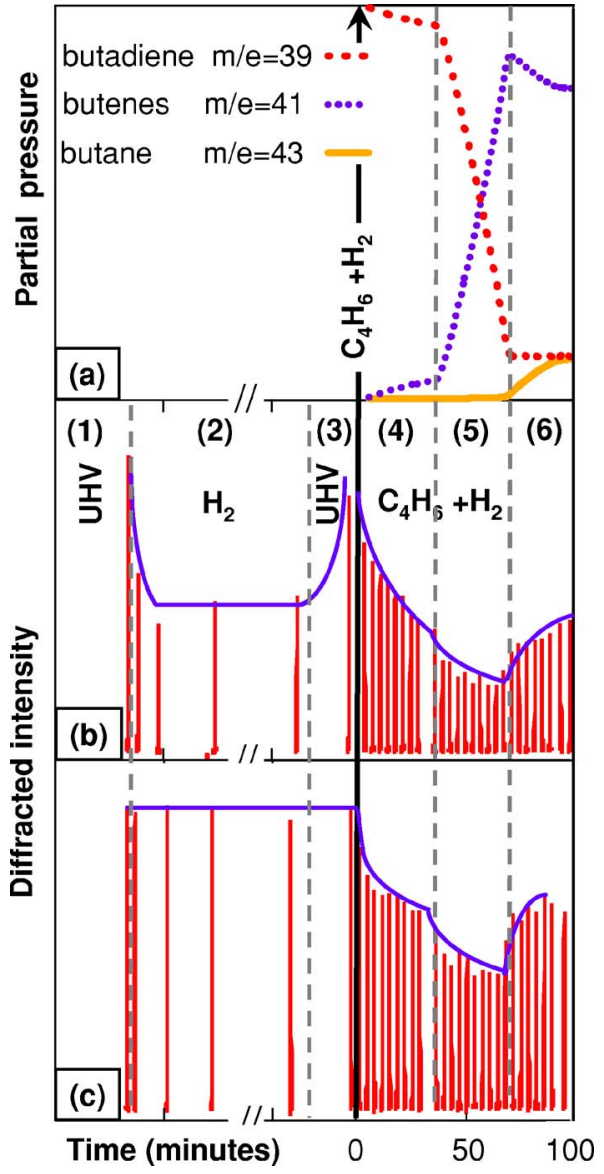

FIG. 10. (Color online) (a) 1,3-butadiene hydrogenation monitored by mass spectrometry. [(b) and (c)] Diffracted intensity evolution during the reaction at $\left(\begin{array}{lll}0 & 1 & 0.03\end{array}\right)$ and $\left(\begin{array}{lll}1 & 0 & 0.03\end{array}\right)$, respectively. The time scale is the same for the three figures; the $Y$ scale was normalized to the butadiene initial value for the partial pressure in (a) and to the UHV value for each diffracted intensity plot in (b) and (c). In region 1 the sample is cleaned in UHV, in region 2 it is exposed to 10 mbars, of hydrogen (during the exposure CTR data reported in Fig. 9 are recorded), and in 3 the reactor chamber was pumped down to UHV. The black vertical arrow indicates the moment when the reactive mixture is introduced, regions 4 and 5 correspond to the butadiene conversion into butenes at $300 \mathrm{~K}$ and then at $500 \mathrm{~K}$, respectively. In region 6 the butene conversion into butane takes place.

slow but was significantly accelerated by increasing the sample temperature from room temperature $(300 \mathrm{~K})$ up to $500 \mathrm{~K}$. Once the butadiene was completely converted into butenes, the hydrogenation of butenes to butane starts.

Figures 10(b) and 10(c) show the diffracted intensities recorded simultaneously at $\left(\begin{array}{llll}0 & 1 & 0.03\end{array}\right)$ and at $\left(\begin{array}{lll}1 & 0 & 0.03\end{array}\right)$. The first values (region 2) correspond to the exposure to pure hydrogen, before and after the CTR recording for the structure determination described just before. It can be seen in Fig. 10(b) that the intensity at $\left(\begin{array}{lll}0 & 1 & 0.03\end{array}\right)$ is reduced by about $40^{\circ} \%$ compared to its UHV value (region 1) as soon as hydrogen is introduced and it stays constant all the time during CTR measurement. Afterwards, it recovers its UHV value when hydrogen is evacuated (region 3). On the contrary the $\left(\begin{array}{lll}1 & 0 & 0.03\end{array}\right)$ intensity is not influenced by the hydrogen pressure as shown in Fig. 10(c).

The reactive mixture introduction (region 4) induces a small intensity drop of both diffraction peaks followed by a gentle decrease during the first hydrogenation step (conver- 
sion of butadiene into butenes). This behavior is almost not modified when the sample temperature is increased up to $470 \mathrm{~K}$ to speed up the reaction (region 5). Once the butadiene is totally transformed, the surface diffraction peak intensities start to slowly increase while the second part of the reaction, butene hydrogenation into butane, is in progress (region 6).

\section{Discussion}

The present results for the $\left(\begin{array}{lll}0 & 1 & 0.03\end{array}\right)$ surface peak are consistent with those of the previously published work. ${ }^{7}$ However, since it was possible to record more data at the same time, the present experiment gives a more precise description of the $\mathrm{Pd}_{8} \mathrm{Ni}_{92}(110)$ surface during the butadiene hydrogenation. First, and quite significantly, the surface appears to be clearly not the same under pure hydrogen as under the reactive mixture. The correlation between the diffracted intensities and the two steps of the reaction evidences that the catalyst surface atoms interact differently with the reactive molecules during the two steps. Indeed, the selectivity of this surface with respect to butenes, defined by the ratio [butenes/[butenes + butane]], is high. It depends on the relative adsorption coefficient of butadiene and butenes on the surface in reaction conditions. It is higher on palladium for butadiene than for butenes. ${ }^{18}$ Thus, as long as butadiene is present in the gas phase, it prevents butane formation by removing butenes from the active sites at the surface. $^{19}$

It was checked that on a sputtered unannealed $\mathrm{Pd}_{8} \mathrm{Ni}_{92}(110)$ surface, which means disordered and without $\mathrm{Pd}$ segregation, no significant reaction occurred under the same butadiene and hydrogen pressures. This stresses that the strong enhancement of the activity of the alloy surface compared to pure $\mathrm{Pd}$ (more than one order of magnitude ${ }^{19}$ ) is directly linked to the strained palladium atoms. It can be assumed that the amplitude of the diffracted intensity variation is roughly correlated with the strength of the binding between the surface atoms and the gas molecules. Indeed, the curves in Figs. 10(b) and 10(c) show that the intensity loss is less important during the second step of the reaction when butenes are absorbed at the surface compared to the first step. Moreover, as already reported, ${ }^{7}$ the effect of pure butadiene on the diffracted intensity is less important compared to the reactive mixture including hydrogen. This supports the fact that the butadiene absorption mode may be different under a high pressure of hydrogen compared to a free-hydrogen environment.

\section{FURTHER DEVELOPMENT}

A new experimental setup has been designed allowing to follow by SXRD and GISAXS the surface structure under (semi-) realistic reaction conditions of model catalysts elaborated in an UHV environment. It was tested by studying the $\mathrm{Pd}_{8} \mathrm{Ni}_{92}(110)$ surface during the butadiene hydrogenation and under hydrogen pressure. The high accuracy of the sample positioning in the incident $\mathrm{x}$-ray beam allowed to record surface diffraction data at grazing incidence. The sample surface was cleaned in an UHV environment $\left(\mathrm{Ar}^{+}\right.$ sputtering and annealing). The surface structure was then monitored under a few millibars of reactive gases, while the reaction products were followed by mass spectrometry. This experiment evidenced the great sensitivity of the diffracted intensity to the subtle interaction between the surface atoms and the reactant molecules. The efficiency of SXRD for such studies is now well established, the limit coming from the common use of point detector. This means long time in order to record the relevant data to determine the structure and to wholly catch the structural changes. Improvements will be obtained by implementing two dimensional (2D) detector.

More recently new experiments were realized with this setup by adding transfer to the connected UHV preparation chamber. It allowed to successfully perform Auger electron spectroscopy in order to probe the chemical surface composition variation induced by reactive gases. The $\mathrm{Au}_{30} \mathrm{Pd}_{70}(111)$ alloy surface was studied under few millibars of hydrogen and during butadiene hydrogenation; the results will be published elsewhere. Concerning the GISAXS facility, studies in (semi-) realistic conditions of supported gold nanoparticles, directly elaborated in the connected UHV chamber, are under progress and will be the subject of a further publication.

\section{ACKNOWLEDGMENTS}

All the CNRS-SERAS staff are gratefully acknowledged for their help as well as the staff of the BM32 CRG beamline. The authors would also acknowledge S. Ferrer, O. Robach, H. Isern (who worked on the ESRF ID03 beamline during the setup building), Y. Jugnet, (IRCE-Villeurbanne), and M. De Santis (Institut Néel Grenoble) for fruitful discussions. The authors also thank the ESRF and CNRS security offices for their advices concerning the laser beam and the gas manifold installations, as well as ESRF and their committees for beam time allocation. The authors associated to these thank J.-P. Levy who is now retired.

\footnotetext{
${ }^{1}$ See B. L. M. Hendricksen, S. Bobaru, and J. Frenken, Top. Catal. 36, 43 (2005), and references therein.

${ }^{2}$ D. Loffreda, L. Piccolo, and P. Sautet, Phys. Rev. B 71, 113414 (2005).

${ }^{3}$ Y. Jugnet, F. J. Cadete Santos Aires, C. Deranlot, L. Piccolo, and J. C. Bertolini, Surf. Sci. 521, L639 (2002).

${ }^{4}$ T. Dellwig, G. Rupprechter, H. Unterhalt, and H.-J. Freund, Phys. Rev. Lett. 85, 776 (2000).

${ }^{5}$ P. Bernard, K. F. Peters, J. Alvarez, and S. Ferrer, Rev. Sci. Instrum. 70, 1478 (1999).

${ }^{6}$ K. F. Peters, C. J. Walker, P. Steadman, O. Robach, H. Isern, and S. Ferrer, Phys. Rev. Lett. 86, 5325 (2001).

${ }^{7}$ M.-C. Saint-Lager et al., Surf. Sci. 587, 229 (2005).

${ }^{8}$ M. D. Ackermann et al., Phys. Rev. Lett. 95, 255505 (2005).

${ }^{9}$ B. L. M. Hendriksen and J. W. M. Frenken, Phys. Rev. Lett. 89, 046101 (2002).

${ }^{10}$ A. Naudon and D. Thibaudière, J. Appl. Crystallogr. 30, 822 (1997).

${ }^{11}$ G. Renaud et al., Science 300, 1416 (2003).

${ }^{12}$ R. E. Winans, S. Vajda, B. Lee, S. J. Riley, S. Seifert, G. Y. Tikhonov, and N. A. Tomczyk, J. Phys. Chem. B 108, 18105 (2004); B. Lee, S. Seifert, S. J. Riley, G. Tikhonov, N. A. Tomczyk, S. Vajda, and R. E. Winans, J. Chem. Phys. 123, 074701 (2005); G. Renaud, AIP Conf. Proc. 748 (Modern Trends in Physics Research), 63 (2005).

${ }^{13}$ S. Vajda, R. E. Winans, J. W. Elam, B. Lee, M. J. Pellin, S. Seifert, G. Y. Tikhonov, and N. A. Tomczyk, Top. Catal. 39, 161 (2006).

${ }^{14}$ N. Kasper, A. Stierle, P. Nolte, Y. Jin-Phillipp, T. Wagner, D. G. de Oteyza, and H. Dosch, Surf. Sci. 600, 2860 (2006).
} 
${ }^{15} \mathrm{http}: / /$ www.esrf.fr/exp_facilities/BM32/index.htm

${ }^{16}$ K. W. Evans-Lutterodt and M.-T. Tang, J. Appl. Crystallogr. 28, 318 (1995).

${ }^{17}$ C. Kuhrs, M. Swoboda, and W. Weiss, Top. Catal. 15, 13 (2001).

${ }^{18}$ See J. C. Bertolini and Y. Jugnet, in The Chemical Physics of Solid Sur- faces, Surface Alloys and Alloy Surfaces, edited by D. P. Woodruff (Elsevier, New York, 2002), Vol. 10, Chap. 11, p. 404, and references therein.

${ }^{19}$ A. C. Michel, L. Lianos, J. L. Rousset, P. Delichère, N. S. Prakash, J. Massardier, Y. Jugnet, and J. C. Bertolini, Surf. Sci. 416, 288 (1998). 